\title{
OPTICAL PICOSECOND STUDIES OF BACTERIORHODOPSIN CONTAINING A STERICALLY FIXED RETINAL
}

\author{
H.-J. POLLAND a , M.A. FRANZ a , W. ZINTH ${ }^{a}$, W. KAISER ${ }^{a}$, E. KÖLLING ${ }^{b}$ and D. OESTERHELT ${ }^{b}$ \\ "Physik Department der Technischen Universität München and ${ }^{b}$ Max-Planck-Institut für Biochemie, Am Klopferspitz, \\ 8033 Martinsried bei München (F.R.G.)
}

(Received April 3rd, 1984)

Key words: Bacteriorhodopsin; Retinal analogue; Picosecond spectroscopy; Proton pump

The photochemical behaviour of an analogous bacteriorhodopsin (9,12-Ph-BR) which contains the sterically fixed 9,12-phenylretinal has been investigated with picosecond spectroscopy. The following results have been obtained. No ground-state intermediate photoproduct is found in agreement with the previous observation that 9,12-Ph-BR does not exhibit proton pumping under illumination. The excited singlet state has a lifetime of $\tau_{S}=10 \pm 2 \mathrm{ps}$. This lifetime agrees favourably with the value calculated from the radiative lifetime $\tau_{\text {rad }}=6.2 \mathrm{~ns}$ and the fluorescence quantum efficiency of $1.2 \cdot 10^{-3}$. Excited-state absorption occurs which results in fluorescence in the ultraviolet region. These various observations differ drastically from the corresponding findings on bacteriorhodopsin. Most important for an understanding of the differences is the fact that 9,12-phenylretinal does not isomerize in the protein's binding site in contrast to retinal. Our data therefore suggest that the formation of the intermediate $K$ observed in bacteriorhodopsin is accompanied by the all-trans to 13-cis isomerization.

\section{Introduction}

Bacteriorhodopsin, the main membrane protein of Halobacterium halobium, acts as a light-driven proton pump [1]. Bacteriorhodopsin contains alltrans- and 13-cis-retinal $\underline{1}$ (Fig. 1) as a prosthetic group, which form protonated Schiff's bases with lysine-216 of the polypeptide chain in the apoprotein bacterio-opsin. After excitation, the light-adapted chromophore which contains alltrans protonated Schiff's base undergoes a photochemical cycle with significant changes in its absorption properties. Various transient species named $\mathrm{K}, \mathrm{L}, \mathrm{M}, \mathrm{N}$ and $\mathrm{O}$ have been assigned $[2,3]$. The system returns into its initial state within 10 ms. It has been shown that the variations of the

Abbreviation: 9,12-Ph-BR, 9,12-phenylretinal-bacteriorhodopsin.<smiles>CC1=C(/C=C/C(C)=C/C=C/C(C)=C/C=O)C(C)(C)CCC1</smiles>

Retinal (1)<smiles>CC1=C(/C=C/c2ccc(/C(C)=C/C=O)cc2)C(C)(C)CCC1</smiles>

Fig. 1. Retinal 1 and 3-(4-(2-(2,6,6-trimethyl-1-cyclohexenyl-)vinyl-)phenyl)-2-butenal (9,12-phenylretinal) 2 . 
absorption properties are accompanied by specific proton movements and by configurational changes of the protonated Schiff's base, i.e., a reversible all-trans to 13-cis isomerization [4,5]. Limited information exists on the timing of isomerization. There is strong evidence that the intermediates $\mathrm{L}$ and $M$ occur in the 13-cis form [6-10]. Resonance Raman studies [11,12] suggest that even the intermediate $\mathrm{K}$, which is formed within the first $10 \mathrm{ps}$ after excitation, contains a distorted 13-cis conformation of the protonated Schiff's base. Model compounds $[7,13]$ show that the isomerization of the retinal molecule during the photocycle is essential for the proton-pumping activity. Theoretical considerations and calculations [14,15] are in accordance to these observations.

In this paper, we report on investigations of a modified bacteriorhodopsin which contains a retinal analog 2 (Fig. 1) (3-(4-(2-(2,6,6,-trimethyl-1cyclohexenyl-)-vinyl-)-phenyl-)-2-butenal) as a prosthetic group. This analog can be considered as a $(10 s)$-cis fixed retinal molecule, but, in contrast to retinal, it contains an aromatic ring in the polyene side-chain, which connects the 9-methyl group and C-12 of retinal. Therefore, we call it 9,12-phenylretinal. Only the all-trans form but not the 13-cis isomer combines with bacterio-opsin to form 9,12-phenylretinal-bacteriorhodopsin $(9,12$ Ph-BR). Formation of a protonated Schiff's base was proven by resonance Raman measurements in water and deuterated water (Kölling, E., Oesterhelt, D. Alshuth, T. and Stockburger, H., unpublished data). The analog chromoprotein does not exhibit proton pump-activity [13]. No intermediates corresponding to the species $L$ and $M$ were found.

Here, we describe the primary events of 9,12$\mathrm{Ph}-\mathrm{BR}$ measured in the picosecond range. With a lifetime of $10 \mathrm{ps}$, fluorescence occurs from an excited singlet state, but no indication of a ground-state intermediate is found. By analogy, we interpret these findings to mean that the first events in the retinal molecule are rotations of bonds which lead to the 13-cis state in the intermediate $\mathrm{K}$ in the photocycle of bacteriorhodopsin.

\section{Materials and Methods}

Bacteriorhodopsin containing purple membranes were isolated from Halobacterium halobium strain $\mathbf{R}_{1} \mathbf{M}_{1}$ [16] and bacteriorhodopsin converted to the apoprotein bacterio-opsin by treatment with hydroxylamin and light [17]. By irradiation $(\lambda \geqslant$ $320 \mathrm{~nm}$ ), the product retinaloxin was destroyed. 9,12-Ph-BR and, as a reference bacteriorhodopsin were prepared by adding equimolar amounts of compounds $\underline{2}$ and $\underline{1}$, respectively, in about $20 \mu 1$ isopropanol to $6 \mathrm{ml}$ of $80 \mu \mathrm{M}$ bacterio-opsin suspensions. A final concentration corresponding to 6-8 $A$ units $\left(9,12-\mathrm{Ph}-\mathrm{BR}, \epsilon=31000 \mathrm{M}^{-1} \cdot \mathrm{cm}^{-1}\right.$ and bacteriorhodopsin, $\epsilon=63000 \mathrm{M}^{-1} \cdot \mathrm{cm}^{-1}$ ) was adjusted. Picosecond experiments were made on reconstituted bacteriorhodopsin samples and on bacteriorhodopsin samples in the original purple membrane state. Both samples show the same results in picosecond absorption measurements, i.e., the removal of and reconstitution with retinal has no effect on the properties and dynamics of the intermediate $\mathrm{K}$ in the picosecond time scale (Polland H.-J., Zinth, W., Kaiser, W. and Oesterhelt, D., unpublished data).

The picosecond experiments on 9,12-phenylretinal were performed with single, frequency-doubled $(\lambda=526.5 \mathrm{~nm})$ pulses of low repetition rate $(1 / 4$ $\mathrm{Hz}$ ) generated with the help of a mode-locked $\mathrm{Nd}$ : glass-laser system. Pulses at longer wavelengths were generated by different nonlinear processes [18].

The time-resolved fluorescence studies were made with an excitation wavelength of $\lambda_{\mathrm{ex}}=526.5$ $\mathrm{nm}$. The fluorescence at $\lambda \geqslant 570 \mathrm{~nm}$ was measured using a streak camera with a time resolution of approx. 3 ps. The streak curves are corrected for nonlinearities in the detection system.

For the time-resolved excitation and probe experiments, we used exciting pulses at $540 \mathrm{~nm}$ with a duration of $t_{\mathrm{p}} \simeq 3 \mathrm{ps}$, energy of $150 \mathrm{~nJ}$, a beam diameter of $0.15 \mathrm{~mm}$, and a peak intensity of $I<300 \mathrm{MW} / \mathrm{cm}^{2}$. In general, we worked at low irradiance so that only $15 \%$ of the $9,12-\mathrm{Ph}-\mathrm{BR}$ molecules absorbed one photon during the excitation pulse. The interrogating (probing) light pulses at 553 and $675 \mathrm{~nm}$ had intensities of $5 \%$ of the exciting pulses. They had the same polarization and duration as the excitation pulse and traversed the sample with well-defined delay times $t_{\mathrm{D}}$. Transmission changes of the probing pulse through the sample $\left(A_{490} \mathrm{~nm}\right)=-\log _{10} I / I_{0}=0.6$, light path $=1 \mathrm{~mm}$ ) were measured by a highly sensitive 
detection system. Changes in the transmitted pulse energy were registered with an accuracy of about $0.05 \%$.

The absorption spectrum of the sample was recorded before and after each measurement. No irreversible change, indicating denaturation of the material, was observed.

\section{Results}

\section{Absorption and fluorescence spectra}

Fig. 2 shows the absorption and fluorescence spectra of 9,12-Ph-BR. The main absorption band in the visible spectrum has an absorption maximum at $490 \mathrm{~nm}$ and an extinction coefficient of $\epsilon=31000 \mathrm{M}^{-1} \cdot \mathrm{cm}^{-1}$. The band has a half-width of $4500 \mathrm{~cm}^{-1}$ and extends to $600 \mathrm{~nm}$. From the oscillator strength, the radiative lifetime of $\tau_{\text {rad }} \simeq$ $5.2 \cdot 10^{-9} \mathrm{~s}$ is calculated [19]. This value is close to the radiative lifetime of bacteriorhodopsin, $\tau_{\text {rad }}=$ $7.2 \cdot 10^{-9}$ s (Polland, H.-J., Zinth, W., Kaiser, W. and Oesterhelt, D., unpublished data).

The fluorescence spectrum (spectra resolution $=3 \mathrm{~nm}$ ) of 9,12-Ph-BR was obtained by exciting the sample with light from an argon-ion laser at $\lambda=514 \mathrm{~nm}$. The laser intensity at the place of the sample was $2.5 \cdot 10^{-4} \mathrm{~W} / \mathrm{cm}^{2}$. According to Fig. 2, the maximum of the fluorescence band is located at $605 \mathrm{~nm}$. Of special interest is the relatively high fluorescence quantum efficiency of $\eta=1.2 \cdot 10^{-3}$. This value is approx. 15-times larger than the fluorescence quantum efficiency determined for

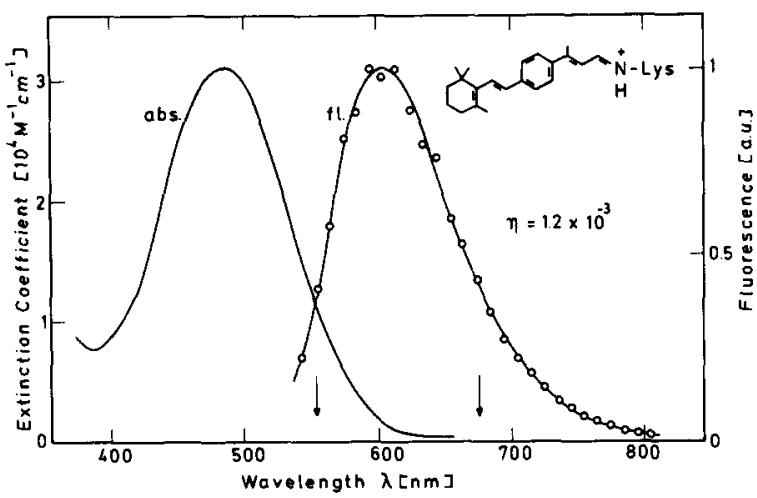

Fig. 2. Absorption spectrum (abs., $\lambda_{\max }=490 \mathrm{~nm}$ ) and fluorescence spectrum (fl., $\lambda_{\max }=605 \mathrm{~nm}$ ) of 9,12-phenylretinal incorporated in bacterio-opsin $(9,12-\mathrm{Ph}-\mathrm{BR})$. The fluorescence quantum yield is $\eta=1.2 \cdot 10^{-3}$. bacteriorhodopsin (Polland, H.-J., Zinth, W. Kaiser, W. and Oesterhelt, D., unpublished data). Since the radiative lifetimes $\tau_{\text {rad }}$ of bacteriorhodopsin and 9,12-Ph-BR are very similar, the higher quantum efficiency of fluorescence in the bacteriorhodopsin analog suggests a longer lifetime $\tau_{\mathrm{S}}$ of the first excited singlet state, $\tau_{\mathrm{S}}=\tau_{\text {rad }} \times$ $\eta \approx 8$ ps. This prediction is confirmed by the fluorescence lifetime measurements described below.

\section{Picosecond measurements}

Fluorescence lifetime of 9,12-Ph-BR. The time dependence of the fluorescence of 9,12-Ph-BR during and after excitation with a light pulse at 526.5 $\mathrm{nm}$ and a duration of $4 \mathrm{ps}$ is shown in Fig. 3. The fluorescence rises rapidly to a peak value during the excitation and decays exponentially with a time-constant $\tau_{\mathrm{S}}=10 \pm 4$ ps. In this experiment, the fluorescence emission with $\lambda \geqslant 570 \mathrm{~nm}$ was measured with a streak camera. The lifetime of 9,12-Ph-BR is markedly longer than that of bacteriorhodopsin analysed under the same conditions. The latter has a lifetime below the time resolution of the streak camera, i.e., the fluorescence lifetime of bacteriorhodopsin is shorter than 3 ps [20] (Polland, H.-J., Zinth, W. Kaiser, W. and Oesterhelt, D., unpublished data).

Transient transmission changes. The transmission changes of 9,12-Ph-BR induced by the excitation at $540 \mathrm{~nm}$ were measured. Data are reported for two significant probing wavelengths. At 675 $\mathrm{nm}, 9,12-\mathrm{Ph}-\mathrm{BR}$ shows little absorption but strong

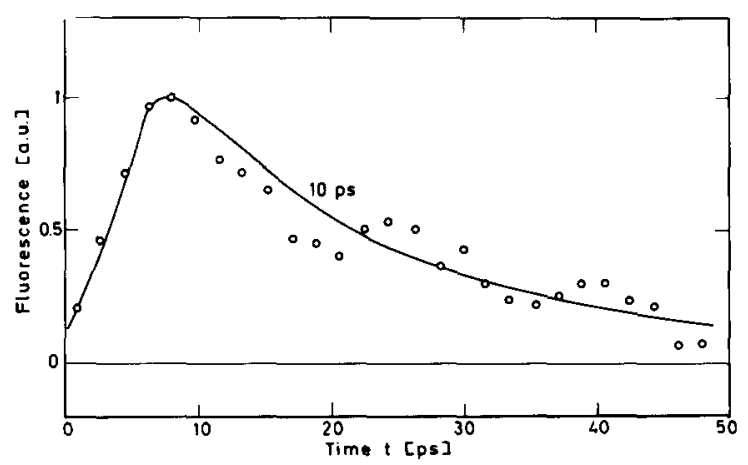

Fig. 3. Time dependence of the fluorescence emission $(\lambda \geqslant 570$ $\mathrm{nm}$ ) from 9,12-Ph-BR excited by an ultrashort laser pulse $\left(t_{\mathrm{p}} \approx 4 \mathrm{ps}\right.$ ) ad $\lambda=526.5 \mathrm{~nm}$. The fluorescence decays to $1 / e$ of the initial value with a time constant of $10 \pm 4$ ps. 
fluorescence, while at $553 \mathrm{~nm}$, the absorption of 9,12-Ph-BR is quite strong (see Fig. 2).

In Fig. 4a, the result with a probing pulse at $675 \mathrm{~nm}$ is presented. The transmitted pulse is stronger (by approx. $1 \%$ at $t_{\mathrm{D}}=1.5 \mathrm{ps}$ ) than the incident pulse, i.e., the probing pulse is amplified. The amplification is caused by stimulated emission due to the transient excess population generated in the first excited singlet state by the excitation pulse. The zero point of the time scale is determined by the coincidence of the peak of the excitation and the probing pulse. The zero point $\left(t_{\mathrm{D}}=0\right)$ and the pulse duration were determined by measuring the cross-correlation curve between excitation and probe pulse (broken line), using a nonlinear optical crystal. According to Fig. 4a, the amplification decreases exponentially with a time constant of $10 \pm 2$ ps. This result points to an excited-state lifetime of 10 ps in full agreement with the fluores-

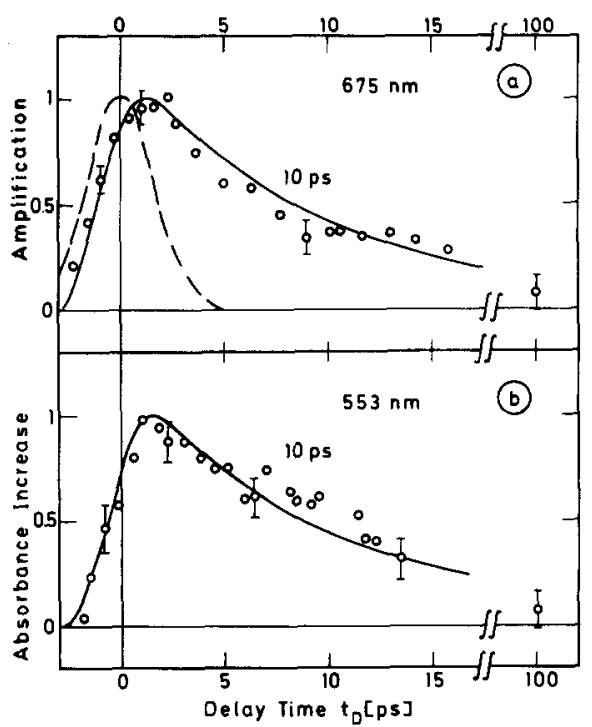

Fig. 4. Picosecond transmission measurements using excitation at $\lambda_{\mathrm{ex}}=540 \mathrm{~nm}$ and probing at $\lambda_{\mathrm{pr}}=675 \mathrm{~nm}$ (a) and $\lambda_{\mathrm{pr}}=553$ $\mathrm{nm}$ (b). Amplification $\left(\log \frac{I_{\mathrm{pr}}\left(t_{\mathrm{D}}\right)}{I_{\mathrm{pr}}(-\infty)}\right)$, part a, and induced absorbance $\left(-\log \frac{I_{\mathrm{pr}}\left(t_{\mathrm{D}}\right)}{I_{\mathrm{pr}}(-\infty)}\right)$, part $\mathrm{b}$, of the probing pulses are shown in arbitrary units. For both probing wavelengths, the same decay time of $10 \pm 2$ ps is observed. There is no indication of a ground-state intermediate. The bars indicate standard deviations which were measured for all delay times but are shown only for selected times. The broken curve is the crosscorrelation curve between excitation and probing pulse. cence data. We note that even for long delay times, $t_{\mathrm{D}} \simeq 100 \mathrm{ps}$, no transient absorption suggesting an intermediate photoproduct was observed.

It should be emphasized that in the experiment with bacteriorhodopsin an absorption for probe pulses at the same wavelength of $675 \mathrm{~nm}$ is observed [21,22] (Poland, H.-J., Zinth, W., Kaiser, W. and Oesterhelt, D., unpublished data). This absorption corresponds to the intermediate $\mathrm{K}$ of bacteriorhodopsin. In 9,12-Ph-BR, no red-shifted ground-state intermediate is found.

At the probe frequency of $553 \mathrm{~nm}$, the monitoring pulse experiences an increased absorbance $\Delta A$ of 0.01 when passing through an excited sample of 9,12-Ph-BR. This result is surprising since, superficially, one would have expected a decreased absorption (bleaching) on account of the increased population of $S_{1}$ and the corresponding depopulation of the ground state. According to Fig. $4 \mathrm{~b}$, the enhanced absorption reaches its maximum at the end of the excitation pulse and decays with a time constant of 10 ps. The increased absorption has the same time dependence as the excess population of the excited singlet state (Fig. 3). This observation suggests that the absorption increase may be due to the transition to higher singlet states. The interpretation is supported by the observation of weak fluorescence emission in the ultraviolet around $300 \mathrm{~nm}$. As in the experiment at $\lambda=675$ $\mathrm{nm}$ for long delay times of $t_{\mathrm{D}} \approx 100 \mathrm{ps}$, we also find no absorption of an intermediate photoproduct at $\lambda=553 \mathrm{~nm}$.

\section{Discussion}

9,12-Phenylretinal in organic solvents can be photoisomerized from the all-trans state to 13-cis and 7-cis isomers [13]. However, a steric hindrance upon all-trans to 13-cis isomerization is introduced by the hydrogen atoms at $\mathrm{C}-11$ and $\mathrm{C}-15$ preventing the molecule from becoming planar. The hindrance can be compensated by a simultaneous rotation of the 12,13-single bond.

If all-trans-9,12-phenylretinal is incorporated into bacterio-opsin, a red-shift of the absorption maximum occurs. The red-shift corresponds to an energy $\left(70.7 \mathrm{~kJ} \cdot \mathrm{mol}^{-1}=5910 \mathrm{~cm}^{-1}\right.$ for the difference between $n$-butylamin Schiff's base and 9,12-Ph-BR) which is even larger than the value 
for retinal and bacteriorhodopsin.

A specific feature of 9,12-phenylretinal is that the 13-cis isomer could neither reconstitute a chromoprotein nor would it be formed in the binding site from the all-trans form in the dark or upon illumination. This can be interpreted as an influence of the protein preventing sterically the compensatory rotation around the 12,13-single bond. Retinal itself in the binding site is isomerized to 13-cis upon excitation and reconverted thermally to all-trans. The 13-cis intermediate is a prerequisite for proton translocation, as was demonstrated by configurationally blocked retinal analogs [7] as well as by the conformationally blocked 9,12-phenylretinal [13]. The latter did neither show activity in proton translocation nor photochemical intermediates in the $\mu$ s range.

The results of our experiments on the picosecond time scale clearly demonstrate that in $9,12-\mathrm{Ph}-$ BR the intermediate analogous to $\mathrm{K}$ is not formed but the molecule returns from the excited state directly to the ground state. At the same time, it seems justified to draw analogies between bacteriorhodopsin and 9,12-Ph-BR regarding the first event after excitation. If no rotation in the region of C-12-C-14 of retinal in bacteriorhodopsin was required for the formation of $K$ then 9,12-Ph-BR would be expected to undergo a corresponding photoreaction leading to the formation of $\mathrm{K}$. Therefore, we assume that the first events in bacteriorhodopsin are such rotations, which in the case of 9,12-Ph-BR are prevented by the protein. This findings are in agreement with the enhanced quantum yield of fluorescence observed in 9,12Ph-BR.

In conclusion, our results give strong evidence that rotational movements of bonds in retinal accompany the first event upon excitation.

\section{Acknowledgements}

We wish to thank Hamamatsu-Television Europe $\mathrm{GmbH}$, Seefeld, for placing the streak camera C 1370-01 at our disposal. This work was supported by the Deutsche Forschungsgemeinschaft (SFB 143).

\section{References}

1 Oesterhelt, D. and Stoeckenius, W. (1973) Proc. Natl. Acad. Sci. USA 70, 2853-2857

2 Lozier, R.H., Bogomolni, R.A. and Stoeckenius, W. (1975) Biophys. J. 15, 955-962

3 Kung, M.C., Devault, D., Hess, B. and Oesterhelt, D. (1975) Biophys. J. 15, 907-911

4 Pettei, M.J., Yudd, A.P., Nakanishi, K., Henselman, R. and Stoeckenius, W. (1977) Biochemistry 16, 1955-1959

5 Aton, B., Doukas, A.G., Callender, R.H., Becher, B. and Ebrey, T.G. (1977) Biochemistry 16, 2995-2999

6 Braiman, M. and Mathies, R. (1980) Biochemistry 19, $5421-5428$

7 Fang, J.-M., Carriker, J.D., Balogh-Nair and Nakanishi, K. (1983) J. Am. Chem. Soc. 105, 5162-5164

8 Stockburger, M., Klusmann, W., Gattermann, H., Massig, G. and Peters, R. (1979) Biochemistry 18, 4886-4900

9 Tsuda, M., Glaccum, M., Nelson, B. and Ebrey, T.G. (1980) Nature 287, 351-353

10 Mowery, P.C. and Stoeckenius, W. (1981) Biochemistry 20, 2302-2306

11 Braiman, M. and Mathies, R. (1982) Proc. Natl. Acad. Sci. USA 79, 403-407

12 Hsieh, C.-L., El-Sayed, M.A., Nicol, M., Nagumo, M. and Lee, J.-H. (1983) Photochem. Photobiol. 38, 83-94

13 Kölling, E., Gärtner, W., Oesterhelt, D. and Ernst, L. (1984) Angew. Chemie Int. Ed. Engl. 23, 81-82

14 Tavan, P., Schulten, K. and Oesterhelt, D. (1984) Biophys. $J$., in the press

15 Schulten, K. and Tavan, P. (1978) Nature 272, 85-86

16 Oesterhelt, D. and Stoeckenius, W. (1974) Methods Enzymol. 31A, 667-678

17 Oesterhelt, D., Schuhmann, L. and Gruber, H. (1974) FEBS Lett. 44, 257-261

18 Polland, H.-J. and Zinth, W. (1984) Opt. Commun. 50, 194-198

19 Strickler, S.J. and Berg, R.A. (1962) J. Chem. Phys. 37, 814-822

20 Sharkov, A.V., Brekhov, O.M. and Rootskoy, B.Y. (1983) Photochem. Photobiol. 38, 109-111

21 Applebury, M.L., Peters, K.S. and Rentzepis, P.M. (1978) Biophys. J. 23, 375-382

22 Shichida, Y., Matuoka, S., Hidaka, Y. and Yoshizawa, T. (1983) Biochim. Biophys. Acta 723, 240-246 